\title{
Comparison of Chromatographic Methods for Determination of the Major Metabolites of Catecholamines and Serotonin
}

\author{
Ilgar S. Mamedov ${ }^{1}$, PhD; Irina V. Zolkina²*, PhD; Pavel B. Glagovsky; \\ Vladimir S. Sukhorukov ${ }^{1}, \mathrm{PhD}, \mathrm{ScD}$ \\ ${ }^{1}$ Russian State Medical University, Moscow, Russia \\ ${ }^{2}$ Research Clinical Institute of Pediatrics, Moscow, Russia
}

\begin{abstract}
In the present article, we set out a new high-tech method for determining the major metabolites of catecholamines and serotonin in urine. We then discuss the effects of these substances and the value of their main metabolites in normal and various pathological conditions, and show the efficiency of applying this technique in the laboratory diagnosis of several diseases.
\end{abstract}

Keywords: catecholamines; serotonin; homovanillic acid; vanillylmandelic acid; 5-hydroxyindolacetic acid; GC-MS.

\section{Introduction}

Catecholamines are a group of aromatic amines, including adrenaline (epinephrine), noradrenaline (norepinephrine), and dopamine, which are synthesized in the adrenal medulla, the sympathetic nervous system, and the brain and act as neurotransmitters and hormones. A significant increase in the level of catecholamines is detected in tumor diseases such as pheochromocytoma, neuroblastoma, and ganglioneuroma, as well as in hypertension, cardiomyopathy, schizophrenia, and manic-depressive disorders [1-4].

Adrenaline and noradrenaline are formed from dopamine and act on the heart muscle, helping the body cope with stress [5,6]. Identification of these compounds is necessary in clinical practice mainly for diagnostic pheochromocytoma (adrenal tissue tumor) and the differential diagnosis of arterial hypertension [3,7]. According to different reviews and statistics, pheochromocytomas account for approximately $0.05 \%$ to $0.6 \%$ of patients with any degree of sustained hypertension [8-10]. Pheochromocytomas are rare catecholamine-secreting tumors that arise from chromaffin tissue within the adrenal medulla and extra-adrenal sites. These neuroendocrine tumors are characterized by an increase in blood catecholamine concentration of 10 to 100 times with preferred secretion of norepinephrine. In essential

*Corresponding author: Irina V. Zolkina, PhD. Research Clinical Institute of Pediatrics, Moscow, Russia.E-mail: izolkina81@gmail.com hypertension, the levels of these compounds in the blood are at the upper limit of normal or increased by 1.5 to 2 times [3]. Furthermore, there is a 10 -fold increase in plasma epinephrine during stress. However, catecholamines are rapidly eliminated from the blood; therefore, for diagnostic purposes it is advisable to examine the urine.

Investigation of catecholamine levels in the blood and urinary excretion is important not only for the diagnosis of the disease, but also to monitor the effectiveness of treatment; thus, the re-increased excretion of catecholamines after radical removal of the tumor may be indicative of its recurrence. Separate determination of epinephrine and norepinephrine in urine allows one to get an indication of the possible location of the tumor: an increased excretion of epinephrine indicates a tumor of the adrenal medulla, and an increased excretion of norepinephrine indicates extra-adrenal tumor. According to the results, the ratio of catecholamines and their metabolites in urine makes it possible to determine the degree of the tumor's "maturation." [7].

A reduced concentration of catecholamines in the urine is marked with a decrease in the filtration capacity of the kidneys, collagenoses, and acute leukemia (especially in children) [11]. Determination of dopamine and the metabolites of epinephrine and norepinephrine is particularly important to confirm the diagnosis of neuroblastoma in children, the third most common neoplasm in older children [12]. The increased excretion of catecholamines and their major metabolites with the urine (homovanillic (HVA) and vanillylmandelic (VMA) acid) is used as a screening test [12]. Among all patients 
examined with an identified neuroblastoma at the early stages, the survival rate was $97 \%$ in contrast to $45 \%$ in children who were diagnosed clinically.

Serotonin is a monoamine neurotransmitter. Approximately $85 \%$ to $90 \%$ of the human body's total serotonin is located in the enterochromaffin cells in the gastrointestinal tract, where it is used to regulate intestinal movements. The remainder is synthesized in serotonergic neurons of the central nervous system, where it has various functions. Serotonin secreted from the enterochromaffin cells eventually finds its way out of tissues into the blood. There, it is actively taken up by blood platelets, which store it. When the platelets bind to a clot, they release serotonin, where it serves as a vasoconstrictor and helps to regulate hemostasis and blood clotting. Serotonin is metabolized mainly to 5-hydroxyindolacetic acid (5HIAA), chiefly by the liver, and excreted by the kidneys. In clinical practice, the determination of serotonin in the blood is particularly informative in carcinoid tumors (a type of neuroendocrine tumor) of the stomach, colon, and lung, when its concentration is increased 5 to 10 times [13]. Determination of elevated levels of the serotonin metabolite 5-HIAA in the urine confirms the presence of a tumor. In typical cases, the diagnosis of the carcinoid syndrome of flushing, which is characterized by short rushes of blood to the face and upper body, diarrhea, development of endocardial fibrosis, is not difficult. But sometimes, in severe hypertension and nonnoticeable other symptoms, it is mistaken for a hypertensive crisis. Such attacks are also observed in mastocytosis, as well as in thyroid cancer in women [14].

Another promising application of a thin chromatographic analysis of metabolites of catecholamines and serotonin is the evaluation of the vegetative status in children. Because, catecholamines, serotonin and their metabolites are sensitive and specific biochemical markers for the diagnosis of various diseases, including childhood, the development of new methods for the analysis of these compounds is important for fundamental medicine as well as for practical medicine.

Methods for determining the levels of metabolites of catecholamines and serotonin in the urine should be very sensitive and accurate, allowing one to identify and trace the dynamics of changes in these values. Such methods of their joint determination as HPLC and GC-MS meet these requirements [15]. For this purpose, ELISA is not sufficiently informative.

The objective of this research was to study the possibility of applying the new algorithm to diagnose various diseases by determining levels of metabolites of catecholamines and serotonin in urine using new high-tech methods and evaluating the effectiveness of the joint determination of these compounds.

\section{Methods}

The study included 100 children aged from 2 to 16 years who were hospitalized in clinical departments (nephrology, genetics, and psycho-neurology) of the Research Clinical Institute of Pediatrics. Written informed consent was obtained from the child's parents.
The study samples were daily urine of patients. After collection of daily urine, about $100 \mathrm{ml}$ from the total urine volume were selected in a plastic container with indication of diuresis. We used $6 \mathrm{~N}$ of hydrochloric acid as the preservative. Samples were frozen at $-20^{\circ} \mathrm{C}$ for prolonged storage, because term storage of samples in a refrigerator at $+2-+8^{\circ} \mathrm{C}$ after acidification cannot exceed 5 days. Selected biomaterial was analyzed by gas and high performance liquid chromatography. Gas chromatography was combined with mass spectrometric detection (GC-MS). Sample preparation processes included selective solvent extraction of catecholamines and serotonin metabolites, and concentration of extractant and derivatization at $80^{\circ} \mathrm{C}$. Methyltestosterone was used as the internal standard.

Determination of metabolites of epinephrine, norepinephrine, serotonin and dopamine (HVA, VMA, and 5-HIAA) was performed by a GC-MS system equipped with an autoinjector AOC--20i (Russian State Medical University, Department of Clinical Laboratory Diagnostics). For each sample, we obtained a chromatogram, in which a concentration of the metabolite in $\mathrm{mg}$ /day was determined via a computer data processing system, CLASS-VPTM Chromatography Data System, for gas chromatography-mass spectrometer. All data were subjected to statistical analysis. A chromatogram of urine obtained by GC-MS is shown in Figure 1.

\section{High performance liquid chromatography (HPLC)}

In the analysis, HVA, VMA, and 5-HIAA were used as the standards. Iso-vanillylmandelic acid served as an internal standard. All compounds were purchased from Sigma-Aldrich. Standard solutions of test compounds at the concentrations of $100 \mathrm{mg} / 1$ were prepared by diluting hydrochloric acid at 10 to $3 \mathrm{M}$ and stored at $4^{\circ} \mathrm{C}$. In this state, they were stable during 6 months. Working solutions were prepared from 10-fold diluted standard solutions.

Analysis of the samples was performed by the liquid chromatography tandem mass spectrometer Agilent Technologies 6410 Triple Quad LC/MS with autosampler, consisting of a double gradient pump Agilent 1200 series, vacuum degasser, and the analytical column Zorbax Eclipse XDB8-C18 (4.0h150 mm; particle size $5 \mathrm{~m}$ ) equipped with a temperature controller.

The data obtained were analyzed using Mass Hunter software for the analysis and identification of the chromatographic peaks. Ultimately, the concentration of a certain metabolite in the urine was calculated in $\mathrm{mg} / \mathrm{day}$. Figure 2 shows the chromatogram of urine obtained by HPLC.

\section{Results}

The high-tech methods of GC-MS and HPLC-MS for the joint determination of metabolites of catecholamines and serotonin in the urine showed the effectiveness of these methods as a diagnostic algorithm, which can be recommended as methods of screening for early detection of a number of neoplastic diseases in children. The methods described in this article are highly sensitive, specific, rapid and not very expensive, which allows them to be used in laboratory practice of medical institutions. 


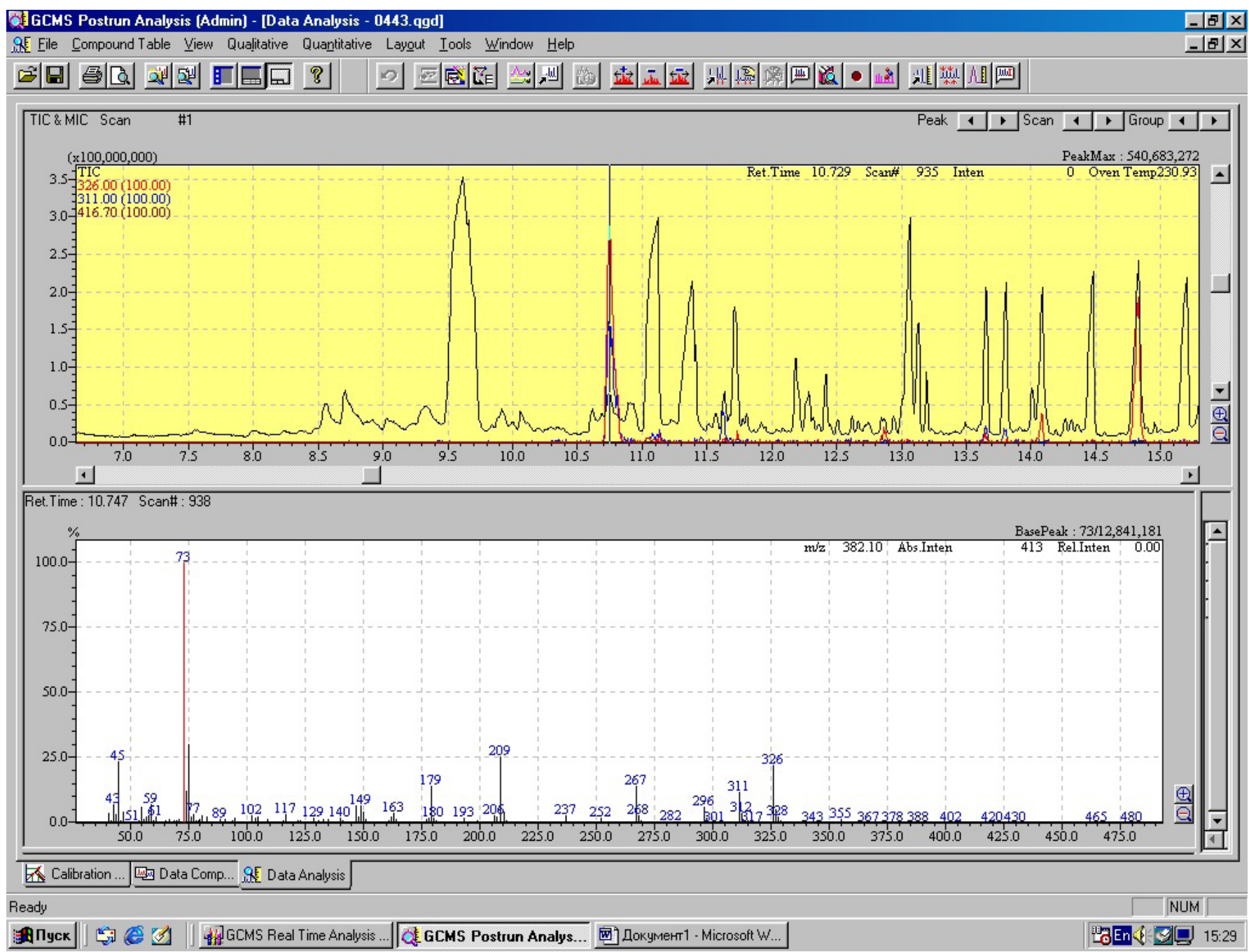

Fig. 1. The chromatogram of urine obtained by GC-MS

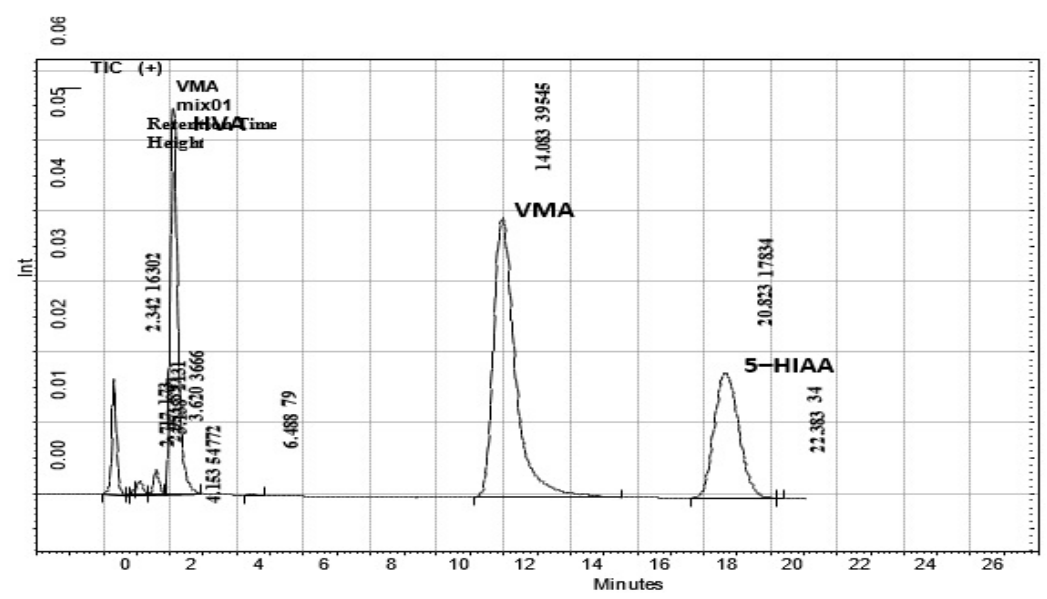

Fig. 2. The chromatogram of urine obtained by HPLC

Chromatographic analysis of the catecholamine and serotonin metabolites was performed in 100 children with suspected various diseases. Based on analysis of the patients' urine, the reference values of catecholamines, serotonin and their main metabolites were determined (Table 1). Diagnosis of neuroblastoma in children was confirmed in 3 cases, when the levels of VMA and HVA in urine were above the age norm, on average 3 times higher. Diagnosis of pheochromocytoma was confirmed in 5 children, and in 3 cases this disease was diagnosed at early stages in the subclinical phase. 
Table 1.

The reference values of catecholamines, serotonin and their major metabolites obtained on the basis of urine analysis by HPLC-MS

\begin{tabular}{|l|c|c|}
\hline \multicolumn{1}{|c|}{ Analytes } & $\begin{array}{c}\text { Value, } \\
\text { nmol/day }\end{array}$ & $\begin{array}{c}\text { Value, } \\
\mu \mathrm{g} / \text { day }\end{array}$ \\
\hline Norepinephrine & $<570$ & $<97$ \\
\hline Epinephrine & $<150$ & $<27$ \\
\hline Dopamine & $<3,240$ & $<500$ \\
\hline VMA & $<33.0$ & $<6.6$ \\
\hline HVA & $<38.0$ & $<6.9$ \\
\hline 5-HIAA & $10.5-47.1$ & $2.0-9.0$ \\
\hline
\end{tabular}

\section{Discussion}

Thanks to the joint research of the Laboratory of General Pathology of RCIP and the Department of Clinical Laboratory Diagnostics of RNRMU, the modern diagnostic algorithm for simultaneous determination of metabolites of catecholamines and serotonin (HVA, VMA, and 5-HIAA) in the urine was designed by GC-MS. It should be noted that the GC-MS method proved to be more convenient for the final processing of results (chromatograms) of patients, because in the software there is an option comparing the obtained peaks with an embedded database of analytes by molecular weight of the substances, which makes the GC-Ms method more accurate and specific, and minimizes the analytical error that can be obtained during the chromatographic separation of substances by liquid chromatography. The results lead us to make a choice in favor of GC-MS, as the most sensitive method. The technique developed reduces analysis time and allows one to analyze up to 100 samples per day. Thus, it is possible to successfully use this method to screen for the presence of neuroblastoma, pheochromocytoma, carcinoid syndrome, and other diseases.

\section{Conclusions}

- A comparative study of the chromatographic methods (HPLC, GC-MS) showed that GC-MS is the most accurate method of determining the major metabolites of catecholamines and serotonin.

- A new modern diagnostic algorithm for jointly identifying the major metabolites of catecholamines and serotonin in urine was developed on the base of GC-MS.

- The reference values of catecholamines, serotonin, and their major metabolites in the urine were determined.

- The study showed that certain levels of the main metabolites of catecholamines and serotonin (HVA, VMA, and 5-HIAA) in the urine could possibly be used as diagnostic markers in clinical and laboratory practice.

\section{Competing interests}

The authors declare that they have no competing interests.

\section{References}

1. Tkachenko BI, Pyatina VF. Human Physiology Compendium. 4th ed. Samara: Samara Printing House, 2002.

2. Kushner B.H. Neuroblastoma: a disease requiring a multitude of imaging studies. J Nucl Med 2004; 45(7):1172-88. 3. Reisch N, Peczkowska M, Januszewicz A, Neumann HP. Pheochromocytoma: presentation, diagnosis and treatment. J Hypertens 2006; 24(12):2331-9.

4. Kjeldsen SE, Neubig RR, Weder AB, Zweifler AJ. The hypertension-coronary heart disease dilemma: the catecholamine-blood platelet connection. J Hypertens 1989; 7(11):851-60.

5. Litschka-Schimpf G, Manzl G, Schimpf A, Weiss M, Eberspächer H, Weicker H. Influence of different experimental recreation treatments on sympathoadrenergic and metabolic regulation mechanisms in repeated exercises. Int J Sports Med 1988; Suppl 2:S146-50.

6. ZumárragaM,DávilaR,BasterrecheN,ArrueA,Goienetxea B, Zamalloa MI, et al. Catechol O-methyltransferase and monoamine oxidase A genotypes, and plasma catecholamine metabolites in bipolar and schizophrenic patients. Neurochem Int 2010; 56(6-7):774-9.

7. Barron J. Phaeochromocytoma: diagnostic challenges for biochemical screening and diagnosis. J Clin Pathol 2010; 63(8):669-674.

8. Manger WM, Gifford RW. Pheochromocytoma. J Clin Hypertens (Greenwich) 2002;4(1):62-72.

9. Bravo EL, Tagle R. Pheochromocytoma: state-of-the-art and future prospects. Endocr Rev 2003;24(4):539-53.

10. Omura M, Saito J, Yamaguchi K, Kakuta Y, Nishikawa T. Prospective study on the prevalence of secondary hypertension among hypertensive patients visiting a general outpatient clinic in Japan. Hypertens Res 2004; 27(3):193-202.

11. Silverstein FS, Hutchinson RJ, Johnston MV. Cerebrospinal fluid biogenic amine metabolites in children during treatment for acute lymphocytic leukemia. Pediatr Res 1986; 20(4):285-91.

12. Izbicki T, Izbicka E, Mazur J. Prognostic significance of biochemical heterogeneity of catecholaminergic clones in neuroblastoma. J Pediatr Surg 2006; 41(9):1506-12.

13. Sukhareva GV, Khomeriki SG. Carcinoid and carcinoid syndrome. Eksp Klin Gastroenterol 2008; 3:110-9. [Article in Russian].

14. Korse CM1, Bonfrer JM, Aaronson NK, Hart AA, Taal BG. Chromogranin $\mathrm{A}$ as an alternative to 5-hydroxyindoleacetic acid in the evaluation of symptoms during treatment of patients with neuroendocrine tumors. Neuroendocrinology 2009; 89(3):296-301.

15. Mamedov IS, Glagovsky PB, Zolkina IV, Sukhorukov VS, Vasin VI. A new method of chromatographic analysis of the metabolites of catecholamines and serotonin in the laboratory diagnosis of various diseases. Vopr Biol Med Farm Khim 2014; 7:59-63. [Article in Russian]. 